\title{
Electron Donor-Acceptor Interaction of 8-Hydroxyquinoline with Citric Acid in Different Solvents: Spectroscopic Studies
}

\author{
Demelash Jado, Khalid Siraj, and Nathan Meka \\ Department of Chemistry, College of Natural Sciences, Jimma University, P.O. Box 378, Jimma, Ethiopia \\ Correspondence should be addressed to Khalid Siraj; chemdocprof@gmail.com
}

Received 15 May 2014; Revised 7 July 2014; Accepted 17 July 2014; Published 17 August 2014

Academic Editor: Ming-Jer Lee

Copyright (C) 2014 Demelash Jado et al. This is an open access article distributed under the Creative Commons Attribution License, which permits unrestricted use, distribution, and reproduction in any medium, provided the original work is properly cited.

\begin{abstract}
Charge transfer complex formation between 8-hydroxyquinoline as the electron donor and citric acid as the electron acceptor has been studied spectrophotometrically in ethanol and methanol solvents at room temperature. Absorption band due to charge transfer complex formation was observed near 320 and $325 \mathrm{~nm}$ in ethanol and methanol, respectively. The stoichiometric ratio of the complex has been found $3: 1$ by using Job's and conductometric titration methods. Benesi-Hildebrand equation has been applied to estimate the formation constant and molecular extinction coefficient. It was found that the value of formation constant was larger in ethanol than in methanol. The physical parameters, ionization potential, and standard free energy change of the formed complex were determined and evaluated in the ethanol and methanol solvents.
\end{abstract}

\section{Introduction}

Mulliken also showed that the charge transfer interactions within a molecular complex consisting of an electron donor (ED) and an electron acceptor (EA) involved a resonance with a transfer of charge from ED to EA $[1,2]$. Charge-transfer complexes result from a donor-acceptor mechanism of Lewis acid-base reaction between two or more different chemical constituents. The formation of electron-donor acceptor (EDA) complexes can be rapidly assessed for its validity as a simple quantitative analytical method for many drug substances which can act as electron donors. Charge transfer complexes have been studied exclusively due to their wide application as ion sensors in the field of environmental science and in the determination of drugs based on the chargetransfer (CT) complexes formed with electron acceptors [35]. They also can be used as organic semiconductors photo catalysts, dendrimers, and in the studying of redox processes [6].

More recently, attention has been given to the isolation and investigation of physical properties of some CTcomplexes in the solid states. Some of these complexes show interesting electrical conductivity properties and have found applications in many forms of electronics and solar cells [7]. Charge transfer complexation is currently to achieve the great importance in biochemical, bioelectrochemical energy transfer process, biological systems, and drug-receptor binding mechanism, for examples, drug action, enzyme catalysis, and ion transfers through lipophilic membranes [8].

Recently, many studies have been widely reported about the rapid interactions between different kinds of drugs and related compounds as donors like morpholine, norfloxacin, ciprofloxacin, and sulfadoxine, with several types of $\sigma$ and $\pi$ electron acceptors $[3,9]$. Charge transfer interactions formed between 8-hydroxyquinoline and acceptors in different solvents has been studied spectrophotometrically [10]. Molecular interactions between electron donors and acceptors are generally associated with the formation of intensely colored charge transfer complexes (CTC) [11].

Organic semiconductors of the CT type can find application as cheap sources for the construction of organic solar batteries in virtue of their semiconducting properties [9]. Charge-transfer complexes are known to take part in many chemical reactions like addition, substitution, and condensation. These complexes have attracted great attention as nonlinear optical materials and electrical conductors. Electron donor-acceptor (EDA) interaction is also important in the field of drug-receptor binding mechanism, in solar energy storage, and in surface chemistry as well as in many biological fields. On the other hand, the EDA reactions 


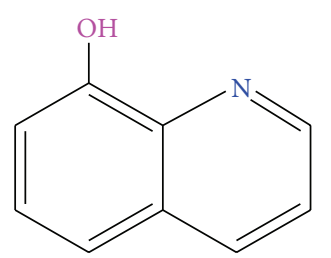

FIGURE 1: Structural illustration of 8-hydroxyquinoline.

of certain $\pi$-acceptors have been successfully utilized in pharmaceutical analysis [12].

8-Hydroxyquinoline is a white to pale yellow crystal or crystalline powder that is insoluble in water or ether and freely soluble in ethanol, acetone, chloroform, benzene, and aqueous mineral acids (Figure 1). It readily forms stable metal chelates which are soluble in organic solvents depending on the $\mathrm{pH}$ of the solution. 8-Hydroxyquinoline is a monoprotic bidentate chelating agent and has been used for the extraction and analytical determination of metal ions due to the ability to coordinate with metal ions. 8-Hydroxyquinoline is an aromatic nitrogen compound characterized by a double-ring structure containing benzene fused to pyridine at two adjacent carbon atoms (pyridine is a ring structure compound of five carbon atoms with a nitrogen atom) [13].

In 8 -HQ the acidic ( $\mathrm{H}$-bond donating) and basic ( $\mathrm{H}$-bond accepting) groups of the molecule are relatively close to each other and hence a single solvent can bind to both sites simultaneously and monomer molecules can also arrange to form dimmers via $\mathrm{H}$-bonding. One might expect tautomerization in H-bond accepting solvents via intramolecular hydrogen bonding and, in all other solvent solutions, there may exist a competition between intra- and intermolecular $\mathrm{H}$-bonding. 8-HQ and its derivatives are capable of forming complexes with many metal ions. 8-HQ has played an important role in organic electroluminescence and was widely introduced in organic electroluminescence cells as emission layer [14].

By considering the above stated applications the present work was designed to carry out spectroscopic studies of electron donor-acceptor interaction of 8-hydroxyquinoline with citric acid in different solvent.

\section{Materials and Method}

2.1. Chemical Used. 8-Hydroxyquinoline (ACS reagent $99 \%$ Sigma Aldrich) and citric acid (Crystal LR 99\% WARDLE CHEMICALS LTD) of the highest purity were used without further purification. Hexane 95\% (UNI-CHEM chemical reagent), ethanol 97\%, and methanol 99.99\% (analytical reagent grade) were used without further purification and also distilled water was used throughout the work.

2.2. Instruments Used. A Shimadzu model 180 UV-VIS double beam spectrophotometer was used for recording the electronic absorption spectra. Hanna Research HI $8820 \mathrm{~N}$ conductivity meter was used for conductance measurements. The ${ }^{1} \mathrm{H}-\mathrm{NMR}$ spectra were recorded at Chemistry Department,
Addis Ababa University, on spectrophotometer using $\mathrm{D}_{2} \mathrm{O}$ (Deuterium oxide) and $\mathrm{CDCl}_{3}$ (Chloroform) as a solvent.

\subsection{Preparation}

2.3.1. Preparation of Standard Solutions. The stock solutions of donor 8-hydroxyquinoline (8-HQ) at concentration of $5 \times$ $10^{-4}$ mole $\mathrm{L}^{-1}$ were freshly prepared by dissolving accurately weighted amounts in ethanol and methanol in appropriate volumetric flask. In the same way the stock solutions of

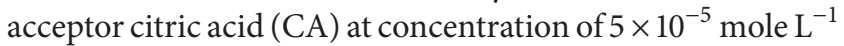
were prepared in different volumetric flasks by dissolving accurately weighted amount in ethanol and methanol solvents.

2.3.2. Synthesis of Complex. The solid CT complex of 8$\mathrm{HQ}$ and citric acid were prepared by mixing $3 \mathrm{mmol}$ of the donor in ethanol/methanol $(40 \mathrm{~mL})$ mixture with $1 \mathrm{mmol}$ of acceptor. The mixture was stirred for 3 hours at room temperature which resulted in the precipitation of the solid CT complexes. The resulting complex was isolated as light yellow crystals. The isolated complex was filtered off using filter paper Whatman No. 1. The isolated solid CT complex was washed well with little amounts of hexane and dried under vacuum.

2.4. Measurement of Conductivity. For conductometric measurement aliquot of $25 \mathrm{~mL}$ of $5 \times 10^{-4} \mathrm{M}$ of 8 -HQ solution was transferred to the beaker and titrated with $5 \times 10^{-5} \mathrm{M}$ of CA solution at room temperature. The conductance values were recorded and plotted versus the titrant volume in ethanol and methanol.

2.5. Solvent Effect. In order to select the suitable solvent for CT complex formation, the reaction of 8-HQ with CA was made in ethanol and methanol solvents. The wavelength of the CT band of the complex and formation constant between 8-HQ and CA was measured in ethanol and methanol solvents.

\section{Result and Discussion}

3.1. Spectral Characteristics of the CT-Complexes. The electronic absorption spectra of the donor, acceptors, and the resulted CT-complexes were carried out in different solvents such as ethanol and methanol in the region of (200-450 nm). Absorption spectra of the reaction product for the complex formed between 8-hydroxyquinoline and citric acid were shown in Figure 2.

Once the donor and acceptor solutions were mixed strong change in color was observed. These changes in colors represent strong evidence of the charge-transfer interactions between the donor and the acceptor. The spectra obtained for CT complex show the new maximum absorption bands at wavelength of $325 \mathrm{~nm}$ and $320 \mathrm{~nm}$ in methanol and ethanol, respectively, which are not due to the absorption of any of the reactants and considered to be the results of CT complex formation between the investigated 8-HQ and CA 


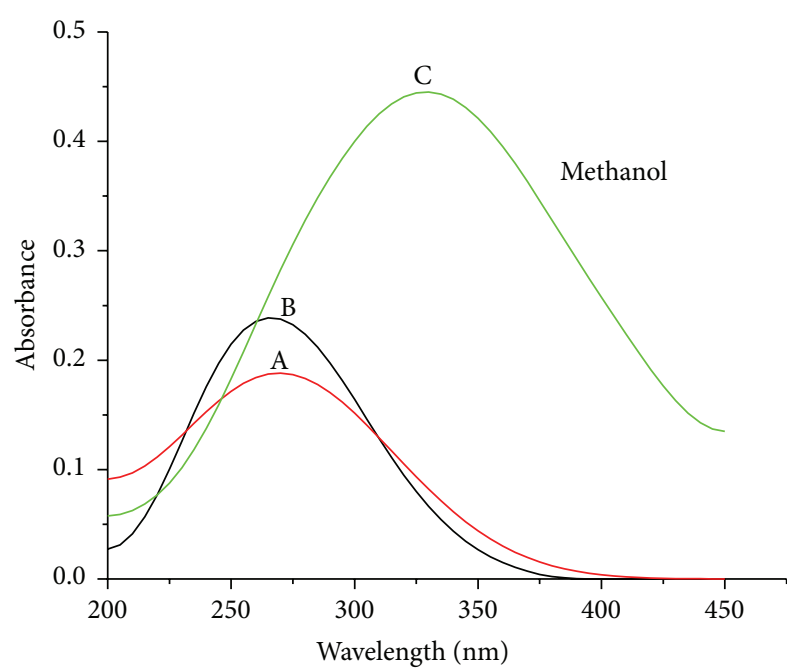

(a)

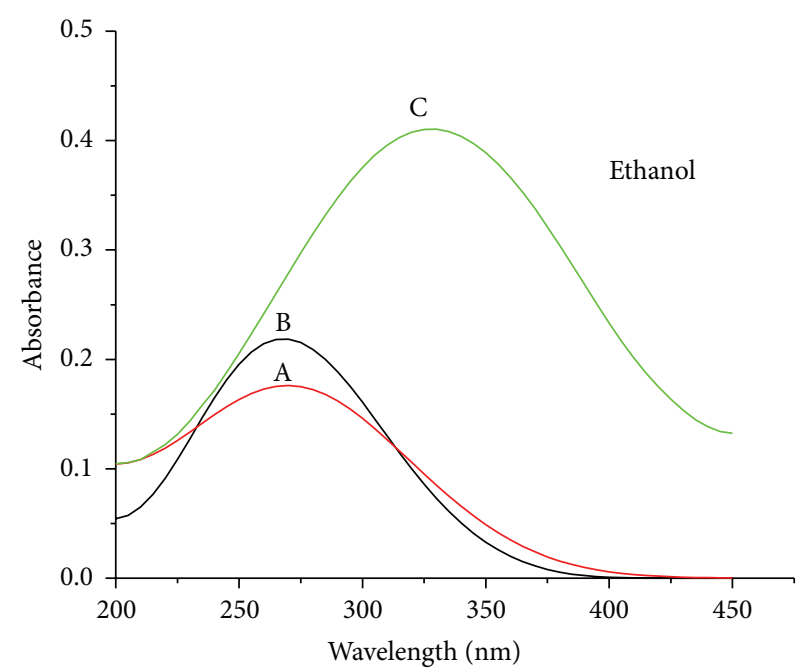

(b)

Figure 2: Electronic spectra of (A) $5 \times 10^{-4} \mathrm{~mol} \mathrm{~L}^{-1} 8$-HQ; (B) $5 \times 10^{-5} \mathrm{~mol} \mathrm{~L}^{-1} \mathrm{CA}$; and (C) CT complex $\left(5 \times 10^{-4} \mathrm{~mol} \mathrm{~L}^{-1} 8-\mathrm{HQ}+5 \times\right.$ $\left.10^{-5} \mathrm{~mol} \mathrm{~L}^{-1} \mathrm{CA}\right)$ in methanol and ethanol.

complex [15]. The new, low energy absorptions observed in solvents containing both a donor and an acceptor have been described by Mulliken as charge transfer transitions involving the excitation of an electron on the donor to an empty orbital on the acceptor [1].

3.2. ${ }^{1} \mathrm{H}-\mathrm{NMR}$ Spectra of CT Complex and Reactants. ${ }^{1} \mathrm{H}-$ NMR spectra of the donor (8-HQ) and the CT complex were measured in $\mathrm{CDCl}_{3}$, whereas the spectrum of an acceptor (CA) was measured in $\mathrm{D}_{2} \mathrm{O}$ using spectrophotometer. The chemical shifts of the different types of protons of the donor, acceptor, and CT complex were interpreted.

The proton transfer from citric acid to the 8-hydroxyquinoline was further confirmed by measuring the ${ }^{1} \mathrm{H}-\mathrm{NMR}$ spectra of the formed complexes. The $4000 \mathrm{MHz}$ nuclear magnetic resonance $\left({ }^{1} \mathrm{H}\right.$-NMR) spectra of the CA and 8$\mathrm{HQ}$ complex were measured in $\mathrm{CDCl}_{3}$ at room temperature. The reaction of CA with 8-HQ yielded a new charge-transfer complex, which produced signals at $\left[(\mathrm{CA})(8-\mathrm{HQ})_{3}\right]: \delta=8.8$ (d, $1 \mathrm{H}, \mathrm{C}-2), 7.5$ (t, $1 \mathrm{H}, \mathrm{C}-3), 8.2$ (d, $1 \mathrm{H}, \mathrm{C}-4), 7.3(\mathrm{~d}, 1 \mathrm{H}, \mathrm{C}-$ 5), $7.4(\mathrm{t}, 1 \mathrm{H}, \mathrm{C}-6)$, and $7.2(\mathrm{~d}, 1 \mathrm{H}, \mathrm{C}-7) 8$ - $\mathrm{HQ}$ ring protons and at $\delta 1.3\left(\mathrm{~s}, 4 \mathrm{H}, \mathrm{CH}_{2}\right)$ of citric acid. It has been found that the phenolic and citric acid protons $(-\mathrm{OH})$ signals, that are observed at $\delta 9.3$ and 10.3 in the spectrum of the free 8-HQ and CA, respectively, disappeared in the spectrum of the CT complex; this indicates the involvement of the $-\mathrm{OH}$ group in chelating through the protonation and deprotonation process from the acceptor to donor migration. These changes in the $\delta(\mathrm{ppm})$ values of the donors and reaction products strongly support the charge migration from the acceptor acid towards the donor base.

3.3. Composition Determination of Charge Transfer Complex. The stoichiometry composition of the CT complex formed in ethanol and methanol was determined by applying Job's

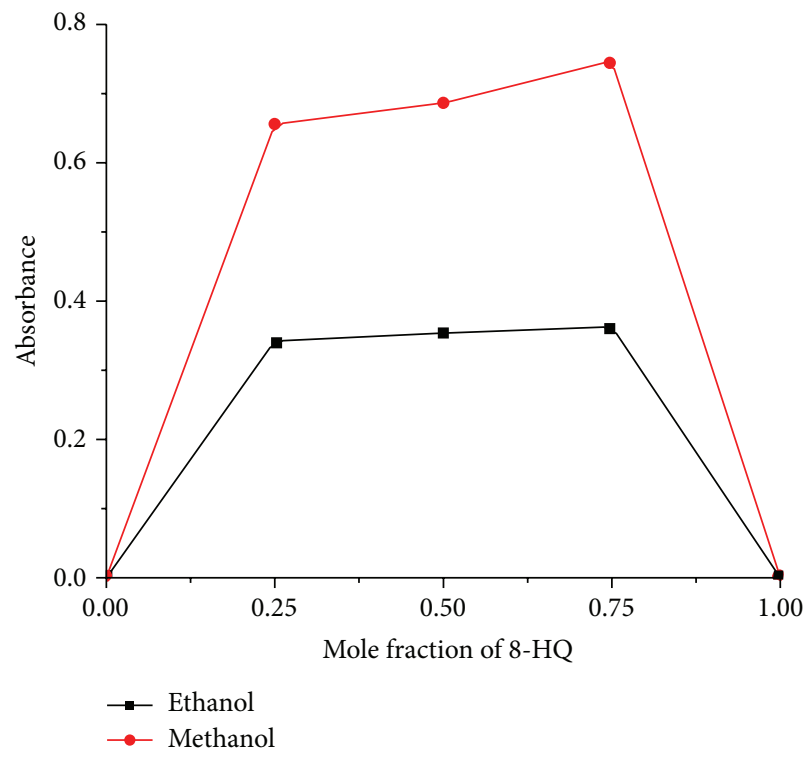

FIGURE 3: Job's method plots of CT complex in ethanol and methanol solvents.

continuous variation method [16]. Figure 3 shows symmetrical curve with a maximum at 0.75 mole fraction indicating the formation of $3: 1$ (D:A) CT complex.

For the conductometric measurement aliquot of $5 \times$ $10^{-4} \mathrm{~mol} \mathrm{~L}^{-1}$ of 8 -HQ solution was transferred to the beaker and titrated with $5 \times 10^{-5} \mathrm{~mol} \mathrm{~L}^{-1}$ of $\mathrm{CA}$ solution at room temperature. Figure 4 shows the conductance values of conductometric measurement recorded and plotted versus the titrant volume in ethanol and methanol.

The resulted complexation solution exhibits appreciable conductance which may be explained by possible formation of charge transfer complex between the reaction partners in 


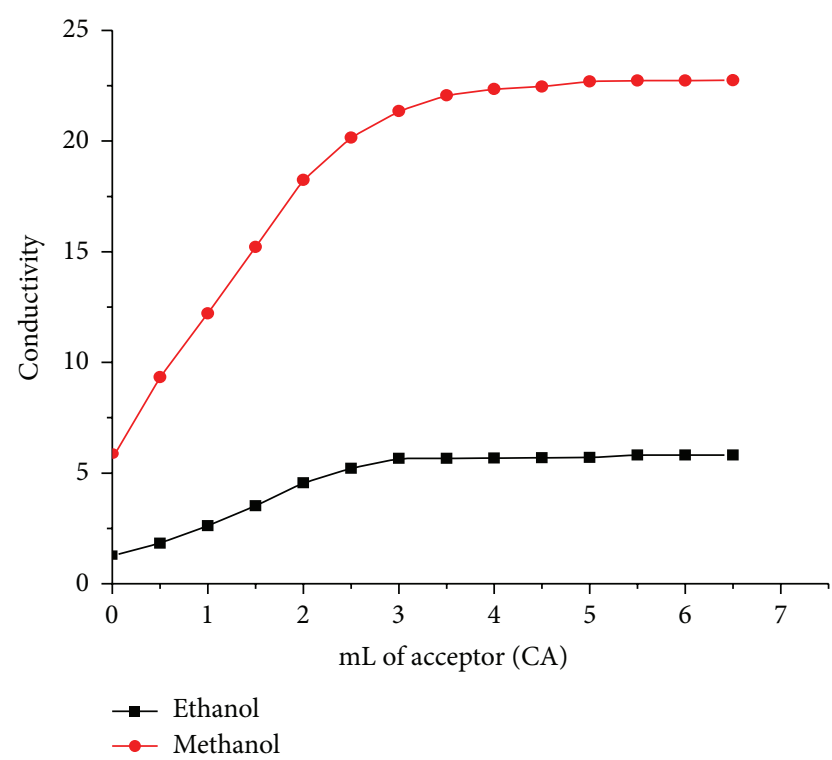

FIGURE 4: Conductometric titration plots of CT complex in ethanol and methanol.

solution. The greater the polarity of the solvent, the greater the formation of ionic species and the conductivity also increases proportionately. It has been observed that the destabilization of the dative structure $\mathrm{D}^{+}-\mathrm{A}^{-}$of CT complex in polar solvent is due to dissociation of the complexes into $\mathrm{D}^{+}$and $\mathrm{A}^{-}[17-$ 19].

3.4. Determination of Formation Constants of the CT Complex. Figure 5 shows the electronic spectra of the intermolecular charge-transfer complex formed from the reactions of 8-HQ with CA at various concentrations of 8-HQ; the values of formation constant $\left(K_{\mathrm{CT}}\right)$ and molecular extinction coefficient $\left(\varepsilon_{\mathrm{CT}}\right)$ were calculated independently under the condition of $[D] \gg[A]$ by using the modified Benesi-Hildebrand equation [20]:

$$
\frac{\left(C_{d}\right)^{3} C_{a}}{A}=\frac{1}{K_{\varepsilon}}+\frac{1}{\varepsilon} C_{d}\left(9 C_{a}+C_{d}\right)
$$

where $C_{a}$ and $C_{d}$ are the initial concentrations of the acceptor and the donor, respectively, and $A$ is the measured absorbance of the detected CT band. It depends on the experimental condition that one of the two component species should be present in large excess. It has been found that the excess of 8-HQ concentration led to a decrease in the complex absorbance. It could be interpreted based on the high steric hindrance of the system at high concentration of 8-HQ that shifts the charge transfer equilibrium towards the reactants [21].

$C_{a} C_{d}^{3} / \mathrm{A}$ values for the $3: 1$ charge-transfer complex is plotted against the corresponding $C_{d}\left(9 C_{a}+C_{d}\right)$ values; a straight line is obtained with a slope of $1 / \varepsilon_{\mathrm{CT}}$ and an intercept of $1 / K \varepsilon_{\mathrm{CT}}$, shown in Figure 6 . From Figure 6 the solvent parameters, $K_{\mathrm{CT}}$ and $\varepsilon_{\mathrm{CT}}$ values associated with the complexes, were calculated and depicted in Table 1.
TABLE 1: Absorption maxima, ionization potential, standard Gibb's free energy change, formation constant, and molar extinction coefficient of CT complex in methanol and ethanol.

\begin{tabular}{lccccc}
\hline Solvent & $\begin{array}{c}\lambda_{\mathrm{CT}} \\
(\mathrm{nm})\end{array}$ & $\begin{array}{c}\mathrm{IP} \\
(\mathrm{ev})\end{array}$ & $\begin{array}{c}\Delta G^{\circ} \\
(\mathrm{kJ} / \mathrm{mol})\end{array}$ & $\begin{array}{c}K_{\mathrm{CT}} \times 10^{-6} \\
\left(\mathrm{~L} \mathrm{~mol}^{-1}\right)\end{array}$ & $\begin{array}{c}\varepsilon_{\mathrm{CT}} \times 10^{-6} \\
\left(\mathrm{~L} \mathrm{~mol}^{-1} \mathrm{~cm}^{-1}\right)\end{array}$ \\
\hline Ethanol & 320 & 10.54 & -43.48 & 7.14 & 2 \\
Methanol & 325 & 10.46 & -33.33 & 6.67 & 5 \\
\hline
\end{tabular}

From Table 1, we can observe the high values of $K_{\mathrm{CT}}$ and $\varepsilon_{\text {CT }}$ in the studied solvents. This confirms the expected high stability of the formed complex in the studied solvents as a result of the high donation power of 8 -HQ. $K_{\mathrm{CT}}$ value for methanol is low as compared to the ethanol which may be due to the solvation of the donor through hydrogen bonding of the nitrogen with $\mathrm{OH}$ of methanol. The high value of $K_{\mathrm{CT}}$ for ethanol can be interpreted as the bulky ethyl group of ethanol compared with methyl group in methanol changes the modes of donor solvation, where hydrogen bonding between the amino group protons and the oxygen of ethanol takes place $[22]$.

3.5. Determination of Ionization Potentials of the Donor. The ionization potential (IP) of the donor of the CT complexes is calculated by using empirical equation derived by Aloisi and Pignataro [23]:

$$
\mathrm{IP}(\mathrm{eV})=5.76+1.53 \times 10^{-4} v_{\mathrm{CT}},
$$

where $v_{\mathrm{CT}}$ is the wave number in $\mathrm{cm}^{-1}$ that corresponds to the CT band formed from interaction between the donor and the acceptor. The electron-donating power of a donor molecule is measured by its ionization potential, which is the energy required to remove an electron from the highest occupied molecular orbital. The ionization potentials of the donor (8-HQ) were 10.54 and 10.46 in ethanol and methanol, respectively (Table 1 ). These values are almost the same in both solvents confirming that IP has limited effect on the stability of the formed complex and solvent independent, but in comparison there is strong interaction between 8HQ and CA in ethanol than methanol. Moreover the same bonding molecular orbital of the donors overlaps with the same antibonding molecular orbital of the acceptors $[22,24]$.

3.6. Determination of Standard Free Energy Changes $\left(\Delta G^{\circ}\right)$. The standard free energy of complexation $\left(\Delta G^{\circ}\right)$ for each complex was calculated from the formation constants using the below equation [25]:

$$
\Delta G^{\circ}=-2.303 R T \log K_{\mathrm{CT}},
$$

where $\Delta G^{\circ}$ is the free energy change $\left(\mathrm{kJmol}^{-1}\right), R$ is the gas constant $\left(8.314 \mathrm{Jmol}^{-1} \mathrm{~K}^{-1}\right), T$ is the temperature in $\mathrm{K}$, and $K_{\mathrm{CT}}$ is the formation constant of the complex $\left(\mathrm{Lmol}^{-1}\right)$ at room temperature. The obtained value of $\Delta \mathrm{G}^{\circ}$ for the CT complex is -33.33 and $-43.48 \mathrm{kJmol}^{-1}$ in methanol and ethanol, respectively; these negative values indicate that the interaction between the donor (8-HQ) and acceptor (CA) is 

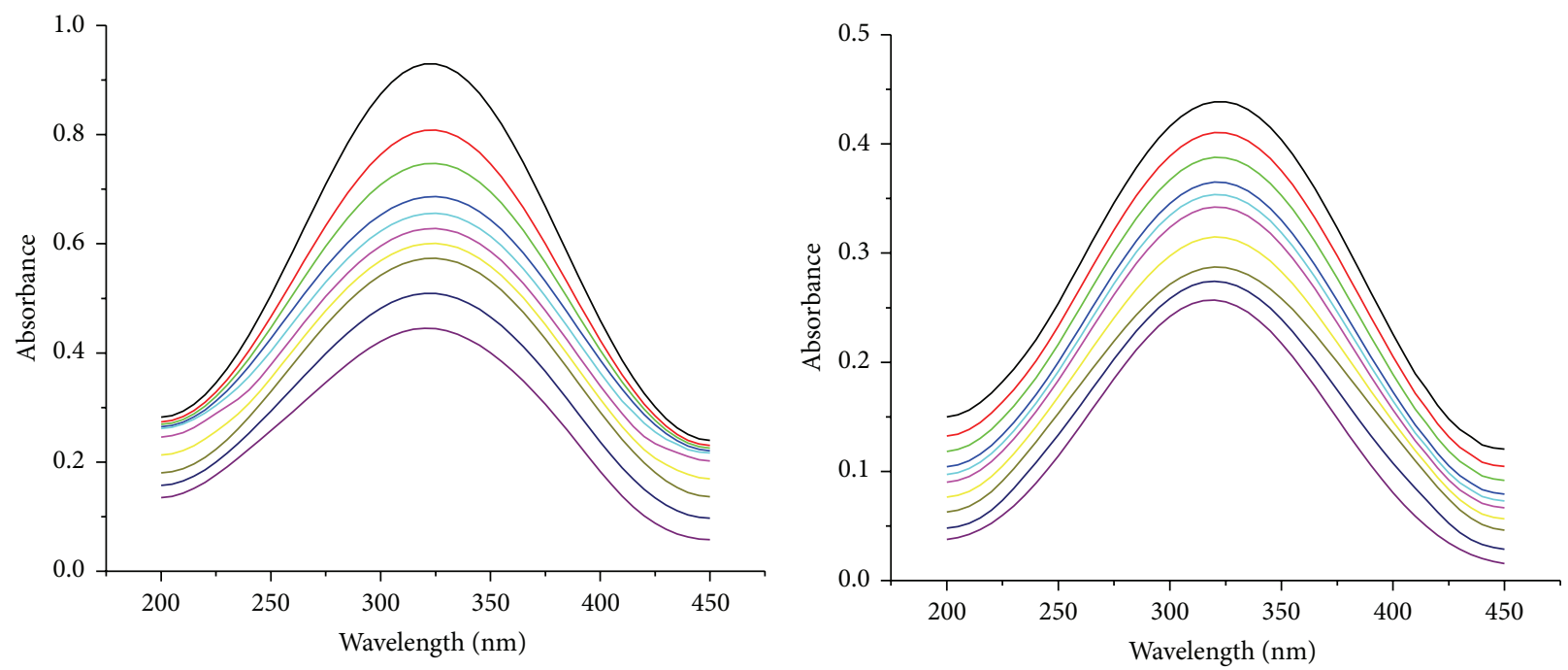

$\begin{array}{ll}\text { Methanol } & \\ -5 \times 10^{-3} & -9 \times 10^{-4} \\ -4 \times 10^{-3} & 8 \times 10^{-4} \\ -3 \times 10^{-3} & -7 \times 10^{-4} \\ -2 \times 10^{-3} & -6 \times 10^{-4} \\ 1 \times 10^{-3} & -5 \times 10^{-4}\end{array}$

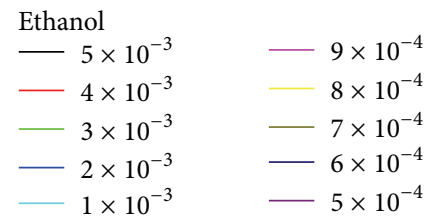

(a)

(b)

Figure 5: Electronic spectra of CT complex recorded for $5 \times 10^{-5} \mathrm{~mol} \mathrm{~L}^{-1} \mathrm{CA}$ with various concentrations $\left(5 \times 10^{-4}\right.$ to $\left.5 \times 10^{-3} \mathrm{~mol} \mathrm{~L}^{-1}\right)$ of 8 -HQ in (a) methanol and (b) ethanol.

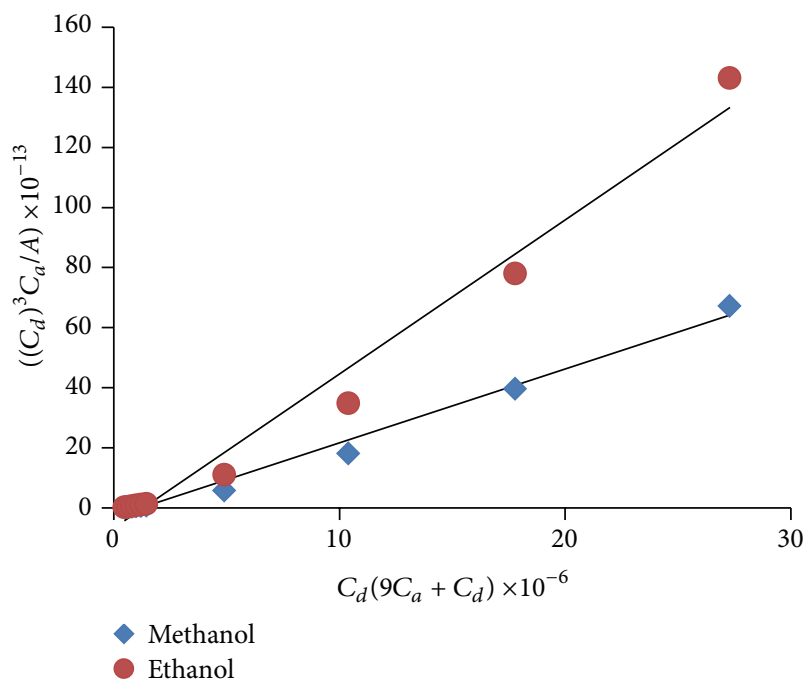

FIGURE 6: Benesi-Hildebrand plots of CT complex in ethanol and methanol solvents.

exothermic and spontaneous (Table 1). The negative values of the free energy change suggest the simultaneous production of the formed complex. The values of $\Delta G^{\circ}$ become more negative as the value of $K_{\mathrm{CT}}$ increases. As the bond between the donor and acceptor becomes stronger and thus the components are subjected to more physical strain or less freedom, the values of $\Delta G^{\circ}$ become more negative [8].
3.7. Effect of Solvent. Absorption spectral characteristics of the CT complexes of studied 8-HQ with CA were carried out in methanol and ethanol solvents at room temperature (Figure 2). However, the most intense absorption was obtained in methanol. Small shift in the position of both lowest and maximum absorption peaks in each spectra in the solvents were found. The greater the polarity of the solvent, the greater the formation of ionic species and the solubility. The experimental results of the CT interaction between 8-HQ and CA in ethanol and methanol solvents show that the values of association constants $K_{\mathrm{CT}}$ value were larger in ethanol than methanol and also the value of molar extinction coefficient of the complex was larger in methanol and smaller in ethanol. This implies that the $K_{\mathrm{CT}}$ values increase from methanol to ethanol with decreasing solvents polarity. Moreover, the increase in $K_{\mathrm{CT}}$ values with decreasing solvents polarity may also be due to the fact that CTC should be stabilized in less polar solvent. It means that the CTC should be strong in less polar solvent than polar solvent. Ethanol was unsuitable solvent for charge transfer complex due to limited solubility. Methanol gave satisfactory results $[7,18]$.

\section{Conclusion}

The charge-transfer complexation reaction of 8-HQ as electron donor and CA as electron acceptors was studied spectrophotometrically in ethanol and methanol at room temperature. A new charge transfer complex was prepared and characterized through infrared, ${ }^{1} \mathrm{HNMR}$, and electronic 
spectra. The stoichiometry of the product was found to be $3: 1$. Benesi-Hildebrand and its modification methods were applied to the determination of formation constant $\left(K_{\mathrm{CT}}\right)$ and molar extinction coefficient $\left(\varepsilon_{\mathrm{CT}}\right)$. Gibb's free energy change and ionization potential of the resulting CT complex was determined. This charge transfer complex would provide information about the synthesis, solvent effect, and electrical conductivity. The result indicates that spectroscopic method would be helpful in improving physical properties.

\section{Conflict of Interests}

The authors declare that there is no conflict of interests regarding the publication of this paper.

\section{Acknowledgment}

The authors would like to thanks to the Department of Chemistry, College of Natural Sciences, Jimma University, Jimma, Ethiopia, for providing necessary facility for carrying out this work.

\section{References}

[1] R. S. Mulliken, "Structures of complexes formed by halogen molecules with aromatic and with oxygenated solvents," Journal of the American Chemical Society, vol. 72, no. 1, pp. 600-608, 1950.

[2] M. S. Refat, M. Y. El-Sayed, A. M. A. Adam, H. A. Saad, and H. H. Eldaroti, "Charge transfer complexes as a semiconductor models: outline of spectroscopic studies on electron donor-acceptor complexes of hexane-1,6-diol with different $\pi$ acceptors," International Journal of Electrochemical Science, vol. 8, no. 3, pp. 4234-4259, 2013.

[3] M. S. Refat, H. A. Didamony, K. M. A. El-Nour, I. Grabchev, L. El-Zayat, and A. M. A. Adam, "Spectroscopic characterizations on the N,N'-bis-alkyl derivatives of 1,4,6,8-naphthalenediimide charge-transfer complexes," Arabian Journal of Chemistry, vol. 4, no. 1, pp. 83-97, 2011.

[4] I. M. Khan, A. Naeem, and A. Ahmad, "Semiquantitative determination of some nitrogen compounds by the formation of charge-transfer complexes of diphenylamine with p-dimethylaminobenzaldehyde by capillary solid-state spottests," Chinese Chemical Letters, vol. 21, no. 6, pp. 720-724, 2010.

[5] D. Wu, L. Zhao, X. Liu et al., "Photon-driven charge transfer and photocatalysis of p-aminothiophenol in metal nanogaps: A DFT study of SERS," Chemical Communications, vol. 47, no. 9, pp. 2520-2522, 2011.

[6] I. M. Khan and A. S. N. Ahmad, "Spectroscopic studies of multiple charge transfer complexes of 8-hydroxyquinoline with $\pi$ acceptor p-Nitrophenol in different solvents at room temperature," Canadian Journal of Analytical Sciences and Spectroscopy, vol. 54, no. 1, pp. 31-37, 2009.

[7] M. S. Refat, S. A. El-Korashy, I. M. El-Deen, and S. M. El-Sayed, "Charge-transfer complexes of sulfamethoxazole drug with different classes of acceptors," Journal of Molecular Structure, vol. 980, no. 1-3, pp. 124-136, 2010.

[8] A. M. A. Adam, M. Salman, T. Sharshar, and M. S. Refat, "Chemical and physical studies on the reaction mechanism of charge-transfer complexes between narcotic drugs and electronic acceptors," International Journal of Electrochemical Science, vol. 8, no. 1, pp. 1274-1294, 2013.

[9] M. S. Refat, L. A. El-Zayat, and O. Z. Yeşilel, "Spectroscopic characterization of charge-transfer complexes of morpholine with chloranilic and picric acids in organic media: crystal structure of bis(morpholinium 2,4,6-trinitrocyclohexanolate)," Spectrochimica Acta A: Molecular and Biomolecular Spectroscopy, vol. 75, no. 2, pp. 745-752, 2010.

[10] I. M. Khan and A. Ahmad, "Spectrophotometric and spectroscopic studies of complexation of 8-hydroxyquinoline with $\pi$ acceptor metadinitrobenzene in different polar solvents," Spectrochimica Acta A: Molecular and Biomolecular Spectroscopy, vol. 73, no. 5, pp. 966-971, 2009.

[11] S. Amirah, M. M. AL-Attas, H. Maram, and T. Basha, "Spectrophotometric and conductimetric studies of charge transfer complexes of some pyrimidine derivatives with chloranilic acid as $\pi$-acceptor in methanol," World Journal of Chemistry, vol. 2, pp. 16-24, 2007.

[12] I. A. Mansour, "Spectral and electrical conductance studies of charge-transfer complexes derived from 6-aminoindazole schiff bases and chloro-p-benzoquinone derivatives," Chemical Papers, vol. 59, no. 3, pp. 174-177, 2005.

[13] F. S. Mooney and J. G. Heathcote, "Lack of proteolytic digestion in the causation of pernicious anaemia," The Lancet, vol. 2, no. 7512, pp. 397-399, 1967.

[14] L. R. Naik and N. N. Math, "Photo physical properties of 8hydroxy quinoline," Indian Journal of Pure and Applied Physics, vol. 43, no. 10, pp. 743-749, 2005.

[15] A. A. Ibrahim, "Spectrophotometric studies of charge transfer complex of 8-hydroxyquinoline with 1, 4-benzoquinone," African Journal of Pure and Applied Chemistry, vol. 5, no. 16, pp. 507-514, 2011.

[16] P. Job, Advanced Physicochemical Experimental, Pitman, London, UK, 1964.

[17] O. M. Al-Shuja'A, A. O. Obeid, and A. G. El-Shekeil, "Spectral, thermal and dc electrical conductivity of charge transfer complex formed between 5,7-dimethyl-1-oxo-2-phenyl-1Hpyrazolo $[1,2-\alpha]$ pyrazol-4- ium-3-olate and iodine," Journal of Macromolecular Science, vol. 48, no. 5, pp. 355-364, 2011.

[18] F. Yakuphanoglu and M. Arslan, "Determination of electrical conduction mechanism and optical band gap of a new charge transfer complex," Solid State Communications, vol. 132, no. 3-4, pp. 229-234, 2004.

[19] F. Doaa, E. H. Baamer, E. L. Mossalamy, S. Salih, and A. L. Juaid, "Spectrophotometric and kinetic studies of charge transfer complexes of pantoprazole with chloranilic acid and DDQ as $\pi$-acceptors," in Proceedings of the 2 nd International Conference on Chemical, Environmental and Biological Sciences, pp. 17-18, 2013.

[20] H. A. Benesi and J. H. Hildebrand, "A spectrophotometric investigation of the interaction of iodine with aromatic hydrocarbons," Journal of the American Chemical Society, vol. 71, no. 8, pp. 2703-2707, 1949.

[21] K. M. Al-Ahmary, M. M. Habeeb, and E. A. Al-Solmy, "Spectroscopic and thermodynamic studies on charge transfer complex formation between 2-aminopyridine and 2,5-dihydroxy- $p$ benzoquinone," Journal of Solution Chemistry, vol. 39, no. 9, pp. 1264-1277, 2010

[22] R. M. Alghanmi, "Spectrophotometric study of charge transfer complex between 2,6-diaminopyridine and 2,5-dihydroxy-pbenzoquinone," Physics and Chemistry of Liquids, vol. 51, no. 3, pp. 365-380, 2013. 
[23] G. G. Aloisi and S. Pignataro, "Molecular complexes of substituted thiophens with $\sigma$ and $\pi$ acceptors. Charge transfer spectra and ionization potentials of the donors," Journal of the Chemical Society, Faraday Transactions 1: Physical Chemistry in Condensed Phases, vol. 69, pp. 534-539, 1973.

[24] A. N. J. Martin and A. Swarbrick, Cammarata, Physical Pharmacy, Lee and Febiger, Philadelphia, Pa, USA, 3rd edition, 1969.

[25] E. Nour, S. Y. Alqaradawi, A. Mostafa, E. Shams, and H. S. Bazzi, "Synthesis, characterization and spectroscopic structural studies of charge-transfer complexes of 1,4,8,11tetraazacyclotetradecane-5,7-dione with iodine, TCNE and DDQ," Journal of Molecular Structure, vol. 980, no. 1-3, pp. 218$224,2010$. 

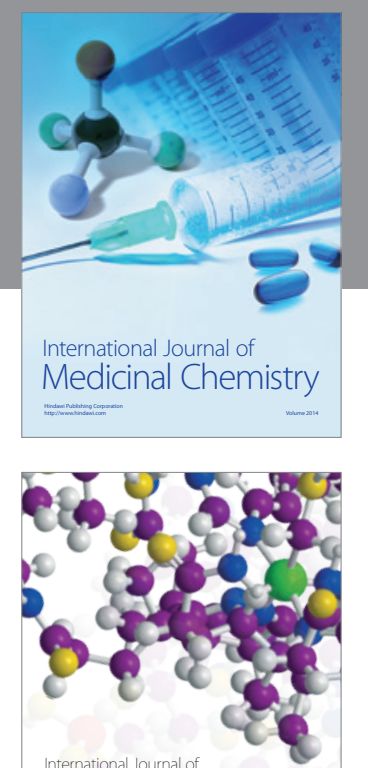

\section{Carbohydrate} Chemistry

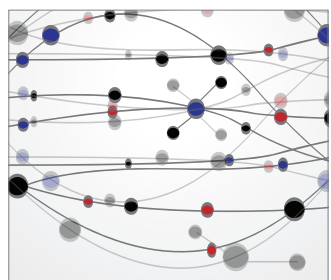

The Scientific World Journal
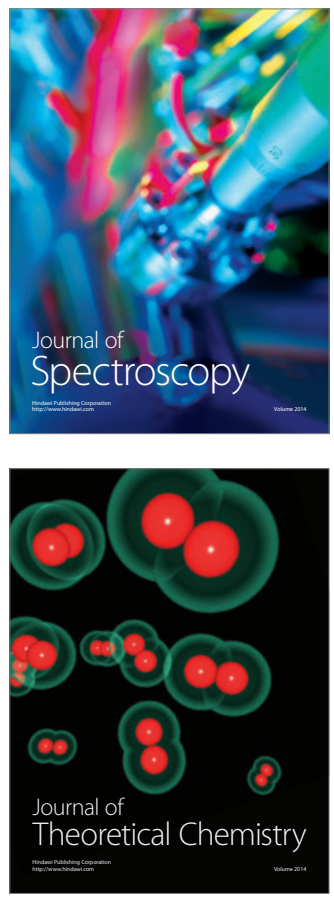
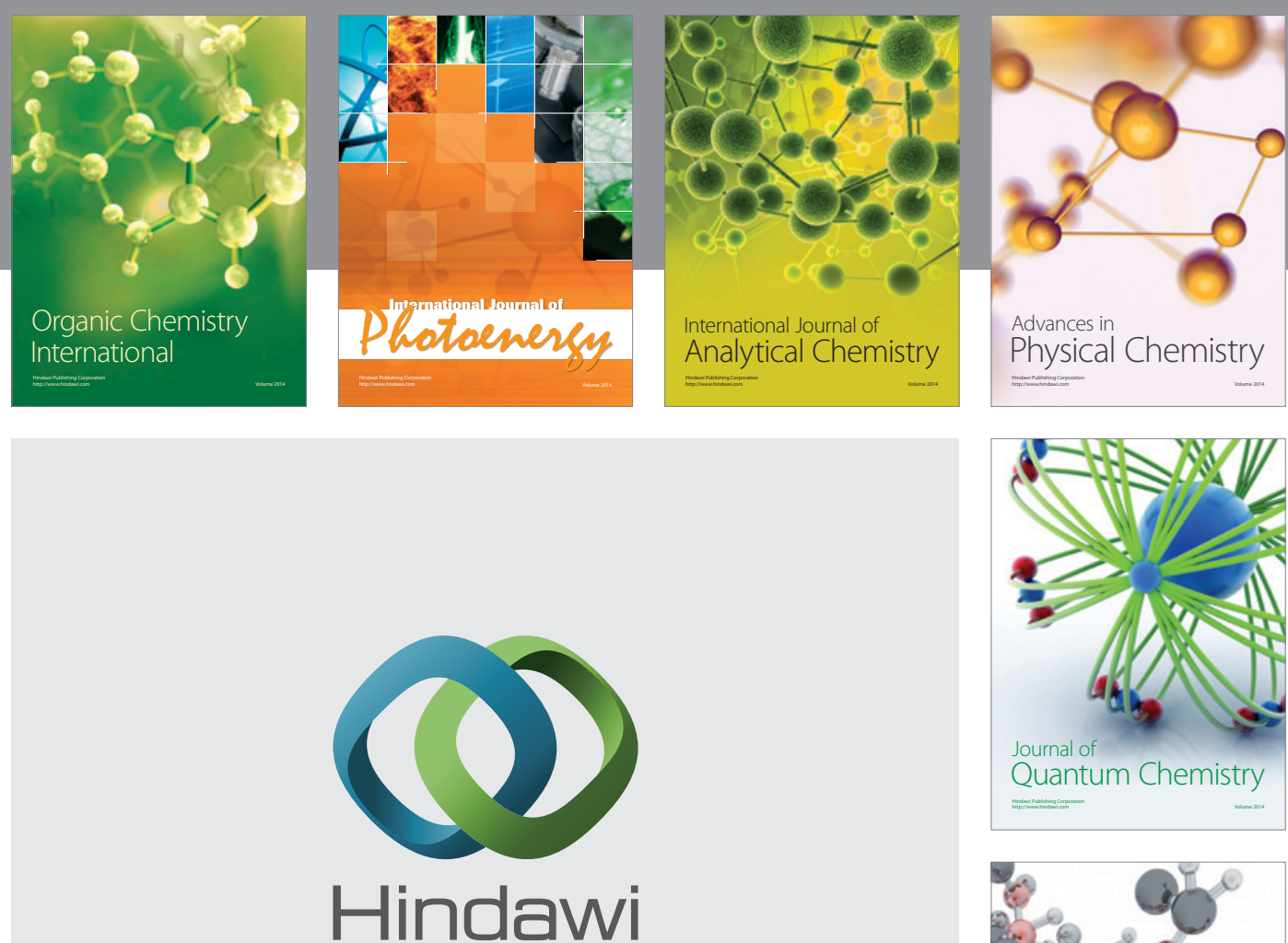

Submit your manuscripts at

http://www.hindawi.com

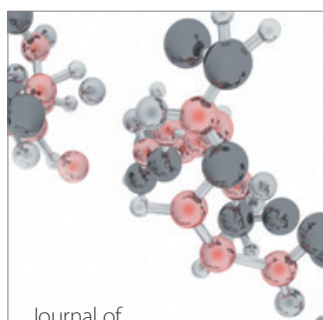

Analytical Methods

in Chemistry

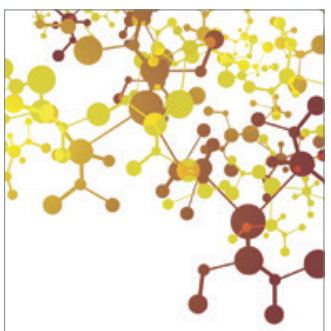

Journal of

Applied Chemistry

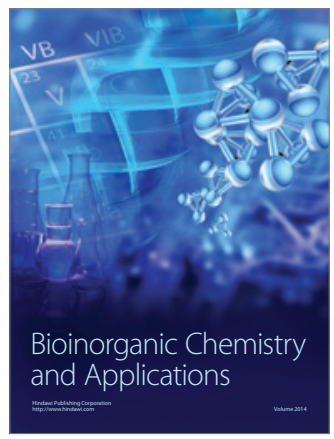

Inorganic Chemistry
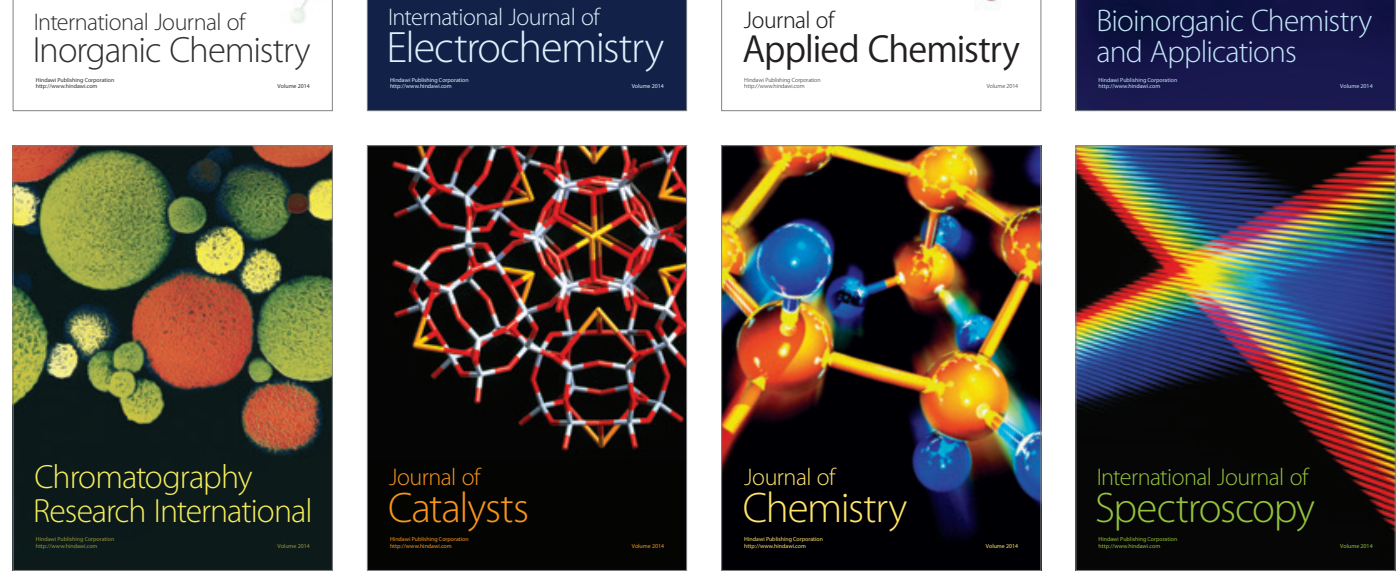